\title{
Facile Synthesis of Gold-Silver Nanocages with Controllable Pores on the Surface
}

\author{
Jingyi Chen ${ }^{\dagger}$, Joseph M. McLellan ${ }^{\dagger}$, Andrew Siekkinen ${ }^{\dagger}$, Yujie Xiong ${ }^{\dagger}$, Zhi-Yuan Li ${ }^{\ddagger}$, and \\ Younan Xia ${ }^{\dagger *}$ \\ $\dagger$ Department of Chemistry, University of Washington, Seattle, Washington 98195-1700 \\ $\$$ Laboratory of Optical Physics, Institute of Physics, Chinese Academy of Sciences, Beijing 100080, P. R. \\ China
}

\section{Abstract}

Gold-silver alloy nanocages with controllable pores on the surface have been synthesized via galvanic replacement reaction between truncated $\mathrm{Ag}$ nanocubes and aqueous $\mathrm{HAuCl}_{4}$. Unlike the previous studies, the initiation of replacement reaction started in a controllable way, simultaneously from eight corners of the truncated Ag nanocubes where $\{111\}$ facets were exposed. The formation of cubic nanocages with pores at all the corners was determined by the capping agent, poly(vinyl pyrrolidone) (PVP), which preferentially covered the $\{100\}$ facets of a truncated Ag nanocube.

Nanocages ${ }^{1}$-- nanostructures with hollow interiors and porous walls -- are useful in applications such as encapsulation and site-specific delivery of chemicals. Nanocages made of noble metals are particularly attractive because of their strong scattering and absorption peaks in the near-infrared region known as surface plasmon resonance (SPR). ${ }^{2}$ Although metallic nanocages can be easily prepared by depositing thin shells of a metal on silica or polymer beads followed by selective removal of the beads, ${ }^{3}$ the polycrystalline walls of such nanostructures often lead to poorly defined pore size/shape and weak mechanical strength. We have recently developed a new method based on galvanic replacement reaction between $\mathrm{Ag}$ nanostructures and $\mathrm{Au}, \mathrm{Pt}$, or Pd compounds to fabricate nanocages characterized by singlecrystal walls. ${ }^{4}$ The same strategy has also been extended by a number of research groups to several other systems. ${ }^{5}$ While small pores often appear on the walls of final product via a dealloying process, they tended to be polydispersed in size/shape and randomly scattered across the surface. Here we demonstrated, for the first time, that the pores can be made uniform in size and shape, and confined to the corners when $\mathrm{Ag}$ nanocubes with truncated corners were used as the sacrificial template.

Figure 1 summarizes all major steps involved in the formation of $\mathrm{Au}-\mathrm{Ag}$ nanocages with wellcontrolled pores at the corners. A key feature of this synthetic procedure is the use of Ag nanocubes whose sharp corners have been truncated in advance via a thermal annealing process. ${ }^{6}$ The mechanism of truncation is in agreement with our previous observation that poly (vinyl pyrrolidone) (PVP, a capping polymer commonly used in the polyol synthesis of $\mathrm{Ag}$ nanostructures) tends to preferentially cover the $\{100\}$ rather than $\{111\}$ facets. ${ }^{7}$ If $\mathrm{Ag}$ nanocubes with sharp corners are thermally aged in ethylene glycol in the presence of a small amount of PVP, $\{111\}$ facets poorly capped by PVP will develop at the corners, leading to truncation of all corners. When added into an aqueous solution of $\mathrm{HAuCl}_{4}$, the corner regions 
unprotected by PVP will serve as primary sites for the dissolution of silver and eventually become well-defined pores at all corners of a nanobox.

As a control experiment, we started with Ag nanocubes bearing sharp corners. Figure 2A shows both SEM and TEM (the inset) images of such nanocubes that were synthesized by introducing a small amount of $\mathrm{HCl}$ (a dual oxidative etchant) to mediate the polyol process. ${ }^{8}$ When these nanocubes with a mean edge length of $94 \pm 14 \mathrm{~nm}$ were mixed with aqueous $\mathrm{HAuCl}_{4}$, the galvanic replacement reaction occurred immediately, following the same mechanism as described in our previous publication. $4 \mathrm{~b}$ More specifically, the reaction was initiated randomly from any one of the six $\{100\}$ faces of a $\mathrm{Ag}$ nanocube through a pitting process (Figure 2B). As the reaction proceeded, nanoboxes (Figure 2C) formed through a combination of replacement and alloying between $\mathrm{Ag}$ and $\mathrm{Au}$. By the end of the reaction, Au nanocages with both hollow interiors and porous walls (Figure 2D) evolved as a result of dealloying of $\mathrm{Ag}$ from the Au-Ag alloyed walls. Although the final structures contained small pores in the surface, the size and shape of these pores were poorly defined and their positions were randomly distributed across the surface.

In comparison, we found that the pores would become more regular and better controlled when Ag nanocubes with truncated corners were employed as the sacrificial template in a similar replacement reaction. To minimize the effect of other factors, we used the same batch of $\mathrm{Ag}$ nanocubes shown in Figure 2A. Their corners were truncated by aging the sample in an ethylene glycol solution containing $1 \mathrm{mM} \mathrm{HCl}$ at $160{ }^{\circ} \mathrm{C}$ for $5 \mathrm{~min}$ in the presence of $0.1 \mathrm{mM} \mathrm{PVP}$ (calculated in terms of the repeating unit). ${ }^{6}$ Because the level of PVP in solution is low, the PVP adsorbed on Ag nanocubes during synthesis will de-adsorb to induce corner truncation. The truncation process can be easily followed by monitoring the change in extinction spectrum. As shown in Figure S1, the number of surface plasmon resonance bands was reduced and the main peak was blue-shifted by $44 \mathrm{~nm}$ after corner truncation at $\{111\}$ facets. These changes could be attributed to the increase in symmetry and slightly reduction in volume as a result of truncation. Figures 2E-2H show SEM and TEM (the inset) images of the nanostructures obtained at different stages of the reaction after the Ag nanocubes with truncated corners had been mixed with aqueous $\mathrm{HAuCl}_{4}$. Different from the cubes with sharp corners, the reaction started simultaneously from all corners of a truncated nanocube while Au atoms were mainly deposited on the side faces (Figure 2F). As a result, cubic nanocages with well-defined pores at all corners (Figure 2G) were obtained as one of the intermediate structures. In the course of this reaction, the ratio of $\{111\}$ to $\{100\}$ facets increased as a result of surface reconstruction. Since the pores are located on the $\{111\}$ facets, their sizes were gradually enlarged until the area of $\{111\}$ facets could not be increased any more. At this point, the product was bound by $\{100\}$ facets with triangular pores at all corners (Figure $2 \mathrm{H}$ ). Depending on the ratio of $\{111\}$ to $\{100\}$ facets, the nanocages might display different orientations on solid supports. As shown in Figure S2, nanocages with minor truncation at the corners tend to lie against the $\{100\}$ planes on a TEM grid while nanocages with major truncation at the corners tend to lie against the $\{111\}$ planes. Continuous addition of $\mathrm{HAuCl}_{4}$ eventually led to fragmentation of such highly porous structures (Figure S3). It is worth pointing out that the nanocages shown in Figures $2 \mathrm{G}$ and $2 \mathrm{H}$ formed as a result of galvanic replacement reaction rather than dealloying process. EDX measurements on the collapsed porous structures shown in Figure S3 indicate that they contained $60 \% \mathrm{Au}$ and $40 \% \mathrm{Ag}$. This composition is different from what was previously observed for nanocages formed through a dealloying mechanism (where it was very close to pure gold).

We also took extinction spectra from the solutions at different stages of the galvanic replacement reaction for both $\mathrm{Ag}$ nanocube samples. As chloroauric acid was added to the sharp nanocubes, the extinction peak was continuously shifted (Figure 3A) from $478 \mathrm{~nm}$ (blue color) to $650 \mathrm{~nm}$ (pink), $770 \mathrm{~nm}$ (red), and $870 \mathrm{~nm}$ (colorless). When the sharp cubes were 
switched to truncated ones, the extinction peak was red-shifted (Figure 3B) from $434 \mathrm{~nm}$ to $650 \mathrm{~nm}$ and $830 \mathrm{~nm}$, when 0.6 and $1.6 \mathrm{~mL} \mathrm{HAuCl}_{4}$ was added to the reaction solution. However, addition of $3.0 \mathrm{~mL} \mathrm{HAuCl}{ }_{4}$ resulted in a blue-shifted, very broad peak. Based on the images shown in Figure $2 \mathrm{H}$, the abnormal shift and broadening were probably related to the broadening in size/shape distribution as caused by surface reconstruction. Although the details of spectral change appear to be different for these two systems, the overall scope of tuning is essentially the same. In addition, our discrete-dipole-approximation (DDA) calculation indicates that the number and location of pores on the surface of a nanocage has a negligible effect on the scattering and absorption cross-sections (Figure S4). As a result, we can make use of the hollow interior and pores associated with the nanocages without compromising their optical properties.

In summary, we have demonstrated a simple way to control the products derived from the galvanic replacement reaction between $\mathrm{Ag}$ nanocubes and aqueous $\mathrm{HAuCl}_{4}$. By controlling the facets exposed on the surface of Ag nanocubes and the facet-selective protection of PVP, we could routinely obtain Au-Ag nanocages containing hollow interiors and controllable pores at all corners. The SPR peaks of these nanostructures could be ready tuned to the near-infrared region to match the transparent window of biological samples. It is expected that such hollow and highly porous nanostructures may find use in controlled release of drugs, optical sensing, and SERS applications. 9

\section{Supplementary Material}

Refer to Web version on PubMed Central for supplementary material.

\section{Acknowledgment}

This work was supported in part by the NIH (R01CA120480), the NSF (DMR-0451788), and a Fellowship from the David and Lucile Packard Foundation. Y.X. is a Camille Dreyfus Teacher Scholar. Z.Y.L. was supported by the National Key Basis Research Special Foundation of China (No. 2004 CB719804). Part of the work was performed at the Nanotech User Facility, a member of the National Nanotechnology Infrastructure Network (NNIN) funded by the NSF.

\section{References}

1. Sun Y, Xia Y. Nano Lett 2003;3:1569.

2. Xia Y, Halas NJ. MRS Bull 2005;30:338.

3. Oldenburg SJ, Averitt RD, Westcott SL, Halas NJ. Chem. Phys. Lett 1998;288:243.

4. (a) Sun Y, Xia Y. Science 2002;298:2176. [PubMed: 12481134] (b) Sun Y, Xia Y. J. Am. Chem. Soc 2004;126:3892. [PubMed: 15038743] (c) Sun Y, Mayers BT, Xia Y. Nano Lett 2002;2:481. (d) Chen J, Wiley B, McLellan J, Xiong Y, Li Z-Y, Xia Y. Nano Lett 2005;5:2058. [PubMed: 16218737]

5. (a) Schwartzberg AM, Olson TY, Talley CE, Zhang JZ. J. Phys. Chem. B 2006;110:19935. [PubMed: 17020380] (b) Yang J, Lee JY, Too H-P. J. Phys. Chem. B 2005;109:19208. [PubMed: 16853479] (c) Vasquez Y, Sra AK, Schaak R. J. Am. Chem. Soc 2005;127:12504. [PubMed: 16144394] (d) Selvakannan PR, Sastry M. Chem. Commun 2005;13:1684. (e) Hao E, Li S, Bailey RC, Zou S, Schatz GC, Hupp JT. J. Phys. Chem. B 2004;108:1224. (f) Métraux GS, Cao YC, Jin R, Mirkin CA. Nano Lett 2003;3:519.

6. McLellan JM, Siekkinen A, Chen J, Xia Y. Chem. Phys. Lett 2006;427:122.

7. Sun Y, Mayers B, Herricks T, Xia Y. Nano Lett 2003;3:955.

8. Im SH, Lee YT, Wiley B, Xia Y. Angew. Chem. Int. Ed 2005;44:2154.

9. (a) Haes AJ, Van Duyne RP. Anal. Bioanal. Chem 2004;379:920. [PubMed: 15338088] (b) Caruso F. Adv. Mater 2001;13:11. (c) Huang H, Remen EE, Kowalewski T, Wooley KL. J. Am. Chem. Soc $1999 ; 121: 3805$. 


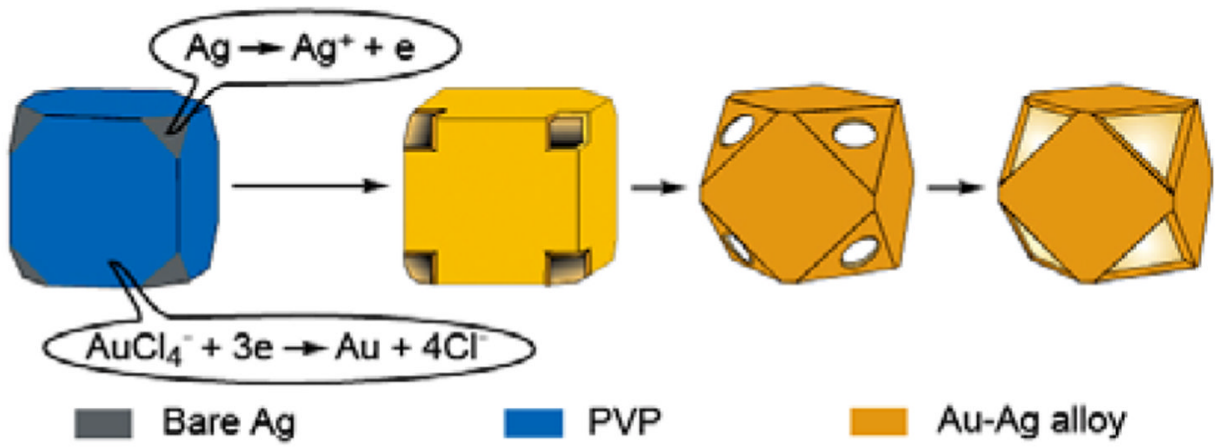

Figure 1.

Schematic illustration detailing all major steps involved in the formation of $\mathrm{Au}-\mathrm{Ag}$ nanocages with well-controlled pores at the corners. The success of this synthesis relies on the use of $\mathrm{Ag}$ nanocubes bearing truncated corners at $\{111\}$ facets to react with aqueous $\mathrm{HAuCl}_{4}$. The pore size is mainly determined by the molar ratio of $\mathrm{HAuCl}_{4}$ to $\mathrm{Ag}$. 

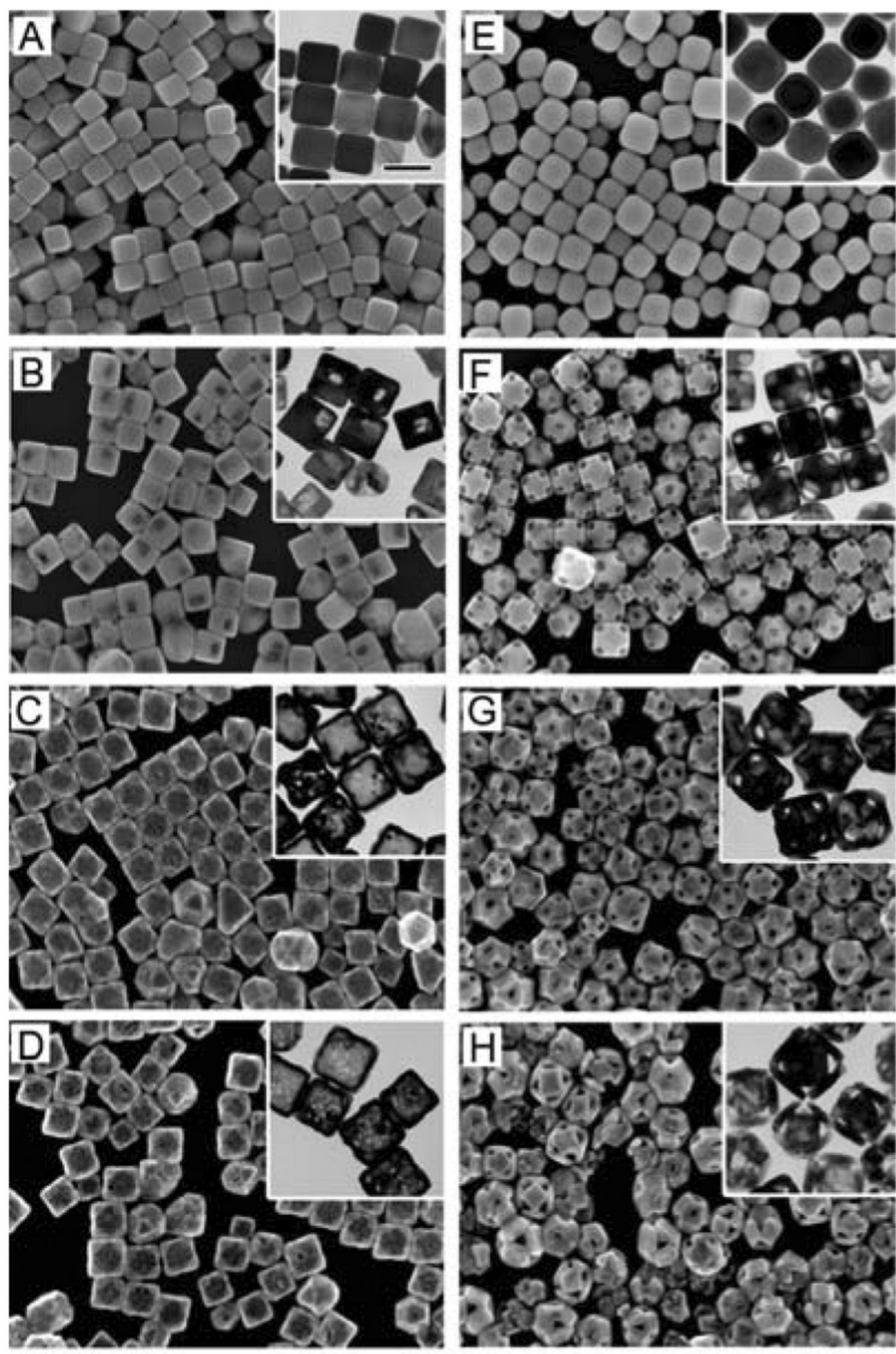

$200 \mathrm{~nm}$

Figure 2.

SEM images of four different stages of galvanic replacement reaction with Ag nanocubes serving as the sacrificial template. (A-D) nanocubes with sharp corners were titrated with different volumes of $0.1 \mathrm{mM} \mathrm{HAuCl}_{4}: 0,0.6,1.6$, and $3.0 \mathrm{~mL}$, respectively. (E-H) Ag nanocubes with truncated corners were reacted with the same volumes of $\mathrm{HAuCl}_{4}$ as for the sharp cubes. The inset shows a TEM image of each sample. The scale bar in the inset is 100 $\mathrm{nm}$, which applies to all TEM images. 

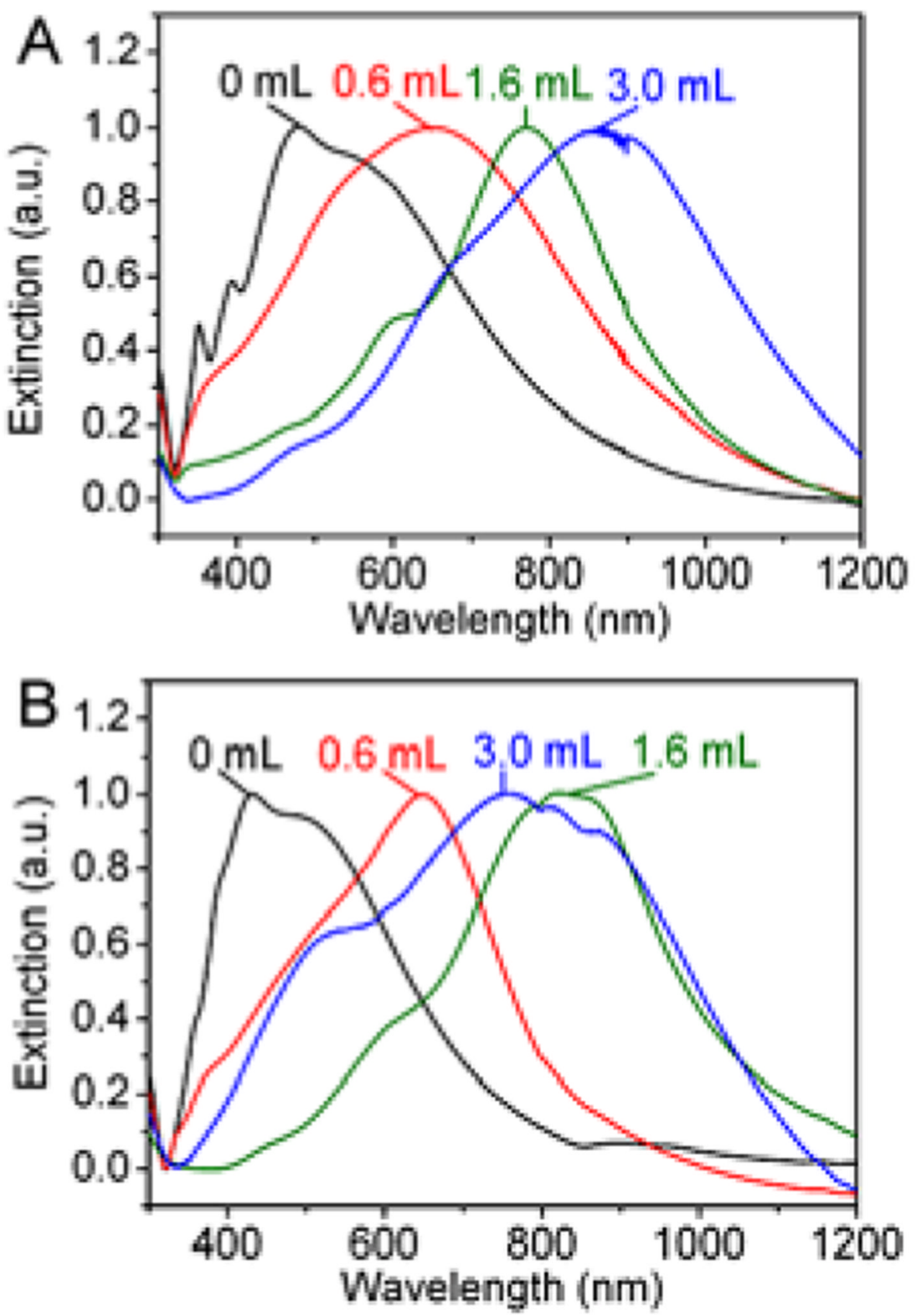

Figure 3.

UV-Vis-NIR spectra of two different types of Ag nanocubes titrated with different volumes of $0.1 \mathrm{mM} \mathrm{HAuCl}_{4}$ : (A) $\mathrm{Ag}$ nanocubes with sharp corners, and (B) Ag nanocubes with truncated corners. 